\title{
The Incidence and Impact of Abdominal Surgery on Delirium in Abdominal Trauma Patients
}

\author{
Hyun Seok Roh, Yun Cheol Park, Young Goun Jo*, Jung Chul Kim \\ Division of Trauma Surgery, Department of Surgery, Chonnam National University Hospital, Gwangju, Korea
}

\section{Article history:}

Received: November 13, 2018

Revised: May 15, 2019

Accepted: October 28, 2019

\begin{abstract}
Purpose: The occurrence of trauma-related delirium following postoperative abdominal surgery is associated with a poor prognosis. The purpose of this study was to identify predictive risk factors for trauma-related delirium.

Methods: Trauma patient data from a regional trauma center were retrospectively collected from August 2015 to December 2016. The primary inclusion criteria were patients diagnosed with traumarelated delirium following abdominal trauma surgery. Head trauma patients and those under 18 years of age were excluded from this study. A multivariate logistic regression analysis was performed to identify the risk factors associated with trauma-related delirium.

Results: Of the 255 trauma patients who met the inclusion criteria, 32 (12.5\%) were diagnosed with delirium. The mean values for the age of the patients, Injury Severity Score, Glasgow Coma Scale score, and length of intensive care unit stay were $52.1 \pm 17.8$ years, $16.9,14$, and 7.1 days, respectively. Among the measured parameters, age [odds ratio (OR), 1.03; 95\% confidence interval (CI), 1.01-1.06; $p=0.022)$ ], sex (OR, 0.125; 0.03-0.55; $p=0.006)$, hemoglobin level (OR, 0.875; CI, 0.68-0.98; $p=0.03$ ), length of stay in the intensive care unit (OR, 1.12; CI, 1.03-1.22; $p=0.01)$, and having an abdominal operation (OR, 2.92; CI, 1.10-7.23; $p=0.011$ ) showed strong correlations with trauma-related delirium
\end{abstract}

Conclusion: This study showed that abdominal surgery was strongly associated with delirium in patients with traumatic abdominal injury. Thus, changes in consciousness should be carefully monitored following surgery.

Keywords: abdominal injuries, critical care, delirium

\section{Hyun Seok Roh}

https://orcid.org/ 0000-0001-8353-8612

Young Goun Jo

https://orcid.org/0000-0002-4418-2729

\section{Introduction}

Delirium occurs in $14 \%$ to $24 \%$ of hospital patients, of which there is a high prevalence (15-53\%) in elderly patients during the post-operative period [1]. Delirium is characterized by an abrupt appearance of symptoms, and its occurrence is common in acute phase trauma patients. Delirium is a syndrome caused by physical and mental changes, and triggers a wide range of symptoms, including decreased brain function. It has been shown that delirium affects the course of treatment and increases mortality rates in postoperative trauma patients $[2,3]$. Delirium is influenced by environmental factors, patient`s characteristics, chronic pathologies and acute illnesses [4]. Age, mechanical ventilation, alcohol use, emergency surgery and trauma are known risk factors associated with this condition $[5,6]$. In trauma patients, delirium is not uncommon, and it increases the duration of hospital and intensive care unit (ICU) stay. It also affects the duration of mechanical ventilation independent to the severity of the trauma $[7,8]$. Understanding the etiology and underlying causes of delirium could lead to a better prognosis through improved prevention and treatment. In this study, we aimed to identify the predictive risk factors for delirium in trauma patients. 


\section{Materials and Methods}

\section{Study design and patient selection criteria}

Between August 2015 and December 2016, patient data were collected retrospectively from a regional trauma center. During the study period, 4,315 injured patients visited the Emergency Department. Of these patients, 817 were major trauma patients with an Injury Severity Score (ISS) > 15 [9]. The primary inclusion criteria were the patients diagnosed with traumarelated delirium following abdominal trauma surgery.

The treatment plan was decided by the trauma surgeon in the emergency room, based on the patient's injured organ, general health status, and any underlying disease. Patients were excluded if they presented without abdominal injuries, were under 18 years of age, or had head injuries.

Conservative treatment was initiated when there was no evidence of hollow organ injuries observed during physical examination, blood test, or other injury signs requiring laparotomy. After the initial treatment [exploratory laparotomy under general anesthesia $(n=99)$ or angioembolization $(n=$ 57)], patients were admitted either to the ICU or to a general ward, depending on their general health status and vital signs. If patients exhibited a change in level of consciousness during the daily ICU surgical rounds, delirium was diagnosed using the Mini Mental State Examination and a psychiatric consultation was requested for evaluation of cognitive function using the Delirium Rating Scale rather than using a delirium diagnostic tool, such as the Confusion Assessment Method for the ICU (CAM-ICU) [10,11].

After diagnosis of delirium by the surgeon, paitents' symptoms were controlled using drugs like dexmedetomidine, and if a symptom was severe, haloperidol or lorazepam were used. After psychiatric consultation, drugs were prescribed which they recommended. Psychiatrists visited the patients to assess the symptom improvement. When a patient's symptoms (including confusion, anxiety) were too severe to wait for psychiatric care, empirical treatment with drugs were used as described above.

This study was approved by the Institutional Review Board at Chonnam National University Hospital (IRB no.: CNUH-2019115).

\section{Statistical analysis}

Continuous data were presented as medians with range, and categorical data were presented as proportions. Continuous variables were compared using the t-test, while proportions were compared using chi-square or Fisher's exact tests, as deemed appropriate. A multivariate logistic regression analysis was performed to identify the risk factors associated with trauma-related delirium. Logistic regression analysis was used to identify significant risk factors. To adjust for confounding factors, variables with a univariate $p<0.05$ were included in the multivariate analysis. A $p<0.05$ was considered to be statistically significant. All statistical analyses were performed using the Statistical Package for the Social Sciences (SPSS Version 21.0, Chicago, IL, USA) for Windows.

\section{Results}

There were 562 of 817 major trauma patients whose data were excluded from the study.

Of the 255 patients who were admitted to ICU, 32 (12.5\%) were diagnosed with delirium. The average age of the patients with delirium was $63.9 \pm 12.9$ years, and 29 (90\%) of them were males. The average ISS value for all patients admitted to ICU was 16.2. Both interventional radiology and surgery were performed to treat abdominal injuries. There were 99 abdominal surgeries performed which included damage control surgery with temporary abdominal closure, bowel resection and anastomosis (small bowel or colon and enterostomy formation), hemostasis (including liver suture hemostasis, omentum ligation, mesentery and internal iliac vessel ligation), splenectomy, gastro-jejunostomy after distal gastrectomy and serosal repair of bowel injury. Regarding sedation and pain control, dexmedetomidine, remifentanil, and midazolam were used, and titrations were performed according to the patients' condition (Table 1).

All patients diagnosed with delirium were admitted to the ICU $(n=32)$ and had on average, longer ICU stays than patients who did not present with delirium ( $7.1 \pm 5.8$ vs. $3.2 \pm 3.5$ days, $p<0.01$ ). The number of ventilator days was also higher in the group diagnosed with delirium $(2.2 \pm 3.3$ vs. $0.6 \pm 2.0$ days, $p<0.016$ ). All deaths occurred within 30 days of admission and during the period of hospitalization. Considering injury severity and occurrence of delirium, the Abbreviated Injury Scale score of abdominal injury was higher in the patients with delirium, but there was no significant difference when comparing thoracic injury and extremity injury, and ISS score (Table 2). Based on univariate analysis, the following factors were associated with the development of delirium: age, sex, hemoglobin level, albumin, abdominal ISS, transfusion, operation, length of ICU stay, ventilator application time and ventilator days, and use of remifentanil and dexmedetomidine.

Multivariate regression analysis showed that age, sex, hemoglobin level, duration of ICU stay, and having abdominal operation are independent risk factors for delirium. Among these, abdominal surgery [odds ratio (OR), 2.92; $95 \%$ confidence interval (CI), 1.199-7.133; $p=0.011)$ ] was observed to be the most significant factor related to the incidence of delirium (Table 3). 
Table 1. Patient characteristics.

\begin{tabular}{|c|c|c|c|}
\hline & \multicolumn{3}{|c|}{ Delirum } \\
\hline & $\begin{array}{l}\text { Present } \\
(n=32)\end{array}$ & $\begin{array}{c}\text { Absent } \\
(n=223)\end{array}$ & $p$ \\
\hline \multicolumn{4}{|l|}{ Patient demographic } \\
\hline Age (y) & $63.9 \pm 12.9$ & $52.1 \pm 17.8$ & $<0.010$ \\
\hline \multicolumn{4}{|l|}{ Sex } \\
\hline Males & $29(15.8)$ & $154(84.2)$ & $<0.010$ \\
\hline Females & $3(4.2)$ & $69(95.8)$ & \\
\hline \multicolumn{4}{|l|}{ Laboratory findings } \\
\hline $\operatorname{WBC}\left(10^{\wedge} 3 / \mathrm{ul}\right)$ & $13.6 \pm 6.4$ & $14.32 \pm 11.2$ & 0.719 \\
\hline $\mathrm{Hgb}(\mathrm{mmHg})$ & $10.4 \pm 2.1$ & $11.7 \pm 2.6$ & $<0.010$ \\
\hline Platelet $\left(10^{\wedge} 3 / \mathrm{ul}\right)$ & $242.1 \pm 259.1$ & $199 \pm 81.7$ & 0.358 \\
\hline Neutrophil (\%) & $77.49 \pm 15.3$ & $77.25 \pm 12.2$ & 0.921 \\
\hline B.E $(\mathrm{mmol} / \mathrm{L})$ & $-4.5 \pm 6.6$ & $-3.5 \pm 5.7$ & 0.341 \\
\hline Albumin (g/dL) & $2.9 \pm 0.6$ & $3.3 \pm 0.7$ & $<0.010$ \\
\hline Lactate $(\mathrm{mmol} / \mathrm{L})$ & $3 \pm 2.0$ & $2.7 \pm 2.7$ & 0.524 \\
\hline \multicolumn{4}{|l|}{ Injury severity } \\
\hline ISS & $17 \pm 5.9$ & $15.6 \pm 8.6$ & 0.244 \\
\hline Chest & $1 \pm 1.4$ & $1.2 \pm 1.4$ & 0.455 \\
\hline Abdomen & $3.2 \pm 0.7$ & $2.8 \pm 0.8$ & 0.018 \\
\hline Extremity & $0.91 \pm 1.42$ & $1.07 \pm 1.4$ & 0.162 \\
\hline Treatment transfusion & & & $<0.010$ \\
\hline Yes & $25(78.1)$ & $118(52.9)$ & \\
\hline No & $7(21.9)$ & $105(47.1)$ & \\
\hline Angioembolization & & & 0.402 \\
\hline Yes & $9(28.1)$ & $48(21.5)$ & \\
\hline No & $23(71.9)$ & $175(78.5)$ & \\
\hline Operation & & & $<0.010$ \\
\hline Yes & $20(62.5)$ & $79(35.4)$ & \\
\hline No & $12(37.5)$ & $144(64.6)$ & \\
\hline \multicolumn{4}{|l|}{ Duration of drug (d) } \\
\hline Remifentanil & $2.9 \pm 4.5$ & $0.6 \pm 2.0$ & $<0.010$ \\
\hline Dexmedetomidine & $1.7 \pm 2.6$ & $0.4 \pm 1.6$ & 0.011 \\
\hline Midazolam & $0.6 \pm 2.3$ & $0.1 \pm 0.6$ & 0.192 \\
\hline
\end{tabular}

Data are presented as $n$ (\%) or mean \pm SD.

$\mathrm{WBC}=$ white blood cell; Hgb = hemoglobin; $\mathrm{B} . \mathrm{E}=$ base excess; ISS = injury severity score.
Table 2. Factors associated with delirium.

\begin{tabular}{lccc}
\hline & \multicolumn{3}{c}{ Delirum } \\
\cline { 2 - 4 } & $\begin{array}{c}\text { Present } \\
(n=32)\end{array}$ & $\begin{array}{c}\text { Absent } \\
(n=223)\end{array}$ & $p$ \\
\hline Clinical course & & 0.011 \\
ICU & & & \\
Yes & & & \\
No & & & \\
Ventilator used & $13(100)$ & & \\
Yes & $19(40.8)$ & $<0.010$ \\
No & & & \\
ICU stay (d) & $7.1 \pm 5.8$ & $3.2 \pm 3.5(15.7)$ & $<0.010$ \\
Ventilator (d) & $2.2 \pm 3.3$ & $0.6 \pm 2.0$ & 0.016 \\
Death & & & 1 \\
Yes & $2(6.2)$ & $17(7.6)$ & \\
No & $30(93.8)$ & $206(92.4)$ & \\
\hline
\end{tabular}

Data are presented as $n(\%)$ or mean \pm SD.

$\mathrm{ICU}=$ intensive care unit.

Table 3. Multivariate regression analysis of risk factors associated with delirium.

\begin{tabular}{lcccc}
\hline \multirow{2}{*}{ Predictor } & \multicolumn{4}{c}{ 95\% Confidence intervals } \\
\cline { 2 - 5 } & Odds ratio & Lower & Higher & $p$ \\
\hline Age $(\mathrm{y})$ & 1.034 & 1.005 & 1.064 & 0.022 \\
Sex & 0.125 & 0.028 & 0.553 & 0.006 \\
$\mathrm{Hgb}(\mathrm{mmHg})$ & 0.875 & 0.681 & 0.980 & 0.030 \\
Operation & 2.924 & 1.199 & 7.133 & 0.018 \\
ICU stay & 1.121 & 1.027 & 1.224 & 0.011 \\
\hline
\end{tabular}

$\mathrm{Hgb}=$ hemoglobin; ICU = intensive care unit.

\section{Discussion}

Delirium is a syndrome that is characterized by an abrupt onset and a fluctuating course, with attention deficit as its core cognitive feature. Changes from a patient's previous baseline are important and show cognitive symptoms such as disorientation, defective memory, and constructional apraxia. Diagnosis of delirium is based on clinical findings such as the patient's medical history, examination of physical and mental status, and a review of medical tests [12]. The occurrence of delirium is the result of multiple factors, of which trauma is a major risk factor. In this current study, age, sex, low hemoglobin level, surgery, and length of ICU stay were observed to be closely related to the incidence of traumarelated delirium. 
Von Rueden et al [13] reported the incidence of delirium among patients admitted to the intermittent care unit, and the ICU. The of delirium prevalence (24\%) was significantly associated with the use of mechanical ventilation, psychotropic medications, high Acute Physiology and Chronic Health Evaluation III scores, and low Richmond Agitation-Sedation Scale scores [13]. In this current study, all patients with delirium were admitted to the ICU, where they had relatively long ICU stays. In ICU, the prevalence of delirium ranges from 20-80\% based on disease severity and the diagnostic method used. In addition to a patient's medical status, uncomfortable ICU environments influence patient outcome [14,15]. In a multicenter study involving 523 ICU-associated cases of delirium, data were collected and analyzed by Van Rompaey et al [4] based on patient characteristics, chronic pathologies, acute illnesses, and environmental factors, and an incidence rate of $30 \%$ was reported.

Consistent with the previous findings, this current study showed that aging and surgery with general anesthesia were closely related to delirium. Bryczkowski et al [16] reported that age was an independent risk factor for delirium in trauma patients who were admitted to the Surgical ICU. The occurrence of delirium in the elderly leads to functional decline, and longer hospitalization and institutionalization, and increases the incidence of complications such as falls and aspiration pneumonia [17]. Post-operative delirium occurs after surgical procedures under general anesthesia, and its incidence has been shown to range from $9 \%$ to $87 \%$ depending on the type of surgery and the age of the patient [18]. Surgery results in a systemic inflammatory response, subsequently affecting the patient's cognitive functions [18].

With the development of radiological diagnostic methods and techniques, it has become possible to establish various treatment plans according to the patient's condition. In the Trauma Division, Department of Surgery, Chonnam National University Medical School, selective non-operative management is performed for solid organ injuries, including liver, spleen, and kidney injuries. Angioembolization of the internal iliac artery after pelvic bone fracture is also performed if the patient's condition is stable [19].

The results in this current study showed that the occurrence of delirium in patients who received interventional radiology was not significantly different from the group that did not receive intervention. Whereas those who underwent surgery were statistically significantly more likely to develop delirium which may be associated with postoperative pain and physiological changes after surgery.

The major limitations of this study related to the screening and diagnosis of the patients. Patients were diagnosed with delirium through the Mini-Mental State Examination and clinical examination, and screened during daily surgical rounds of the ICU. However, diagnostic tools such as CAM-ICU can be a better option in the diagnosis of delirium. The diagnosis and screening approach may have led to the exclusion of relatively hypoactive type patients. Gual et al [20] have reported that the proportion of hypoactive, hyperactive, and mixed type delirium in older vulnerable patients with chronic conditions was $25.9 \%$, $40.6 \%$, and $31 \%$, respectively. In another study, Marcantonio et al [21] reported that the psychomotor types of delirium after hip surgery in the elderly and the pure hypoactive type accounted for $71 \%$ of the patients. Thus, it is likely that in this current study, patients with hypoactive delirium may have been overlooked. Moreover, this study was not a large, multicenter, randomized, retrospective study which may be limiting.

In conclusion, consistent with previously published studies, this study showed that age, being male, and surgery with general anesthesia are associated with delirium. Most importantly, delirium was significantly associated with patients who had abdominal surgery resulting from abdominal traumatic injuries. To address this issue further, careful observation of changes in the level of consciousness of the patient in the post-operative period is necessary, and early diagnosis of this condition followed by the appropriate treatment is recommended.

\section{Conflicts of Interest}

The authors have no conflicts of interest to declare.

\section{References}

[1] Inouye SK. Delirium in older persons. New Eng J Med 2006;354(11):115765.

[2] Lawlor PG, Bush SH. Delirium diagnosis, screening, and management. Curr Opin Support Palliat Care 2014;8(3):286-95.

[3] Meagher DJ, Morandi A, Inouye SK, Ely W, Adamis D, Maclullich AJ, et al. Concordance between DSM-IV and DSM-5 criteria for delirium diagnosis in a pooled database of 768 prospectively evaluated patients using the delirium rating scale-revised-98. BMC Med 2014;12:164.

[4] Van Rompaey B, Elseviers MM, Schuurmans MJ, Shortridge-Baggett LM, Truijen S, Bossaert L. Risk factors for delirium in intensive care patients: A prospective cohort study. Crit Care 2009;13(3):R77.

[5] Zaal IJ, Devlin JW, Peelen LM, Slooter AJ. A systematic review of risk factors for delirium in the ICU. Crit Care Med 2015;43(1):40-7.

[6] Kat MG, de Jonghe JF, Vreeswijk R, van der Ploeg T, van Gool WA, Eikelenboom P, et al. Mortality associated with delirium after hip-surgery: A 2-year follow-up study. Age Ageing 2011;40(3):312-8.

[7] Blondell RD, Powell GE, Dodds HN, Looney SW, Lukan JK. Admission characteristics of trauma patients in whom delirium develops. Am J Surg 2004;187(3):332-7.

[8] Lat I, McMillian W, Taylor S, Janzen JM, Papadopoulos S, Korth L, et al. The impact of delirium on clinical outcomes in mechanically ventilated surgical and trauma patients. Crit Care Med 2009;37(6):1898-905.

[9] Palmer C. Major trauma and the injury severity score--where should we set the bar? Annu Proc Assoc Adv Automot Med 2007;51:13-29.

[10] Soja SL, Pandharipande PP, Fleming SB, Cotton BA, Miller LR, Weaver SG, et al. Implementation, reliability testing, and compliance monitoring of the Confusion Assessment Method for the Intensive Care Unit in trauma patients. Intensive Care Med 2008;34(7):1263-8. 
[11] Grover S, Kate N. Assessment scales for delirium: A review. World J Psychiatry 2012;2(4):58-70.

[12] Neufeld KJ, Thomas C. Delirium: Definition, epidemiology, and diagnosis. J Clin Neurophysiol 2013;30(5):438-42.

[13] Von Rueden KT, Wallizer B, Thurman P, McQuillan K, Andrews T, Merenda J, et al. Delirium in Trauma Patients: Prevalence and Predictors. Crit Care Nurse 2017;37(1):40-8.

[14] Girard TD, Pandharipande PP, Ely EW. Delirium in the intensive care unit. Crit Care 2008;12 Suppl 3:S3.

[15] Reade MC, Finfer S. Sedation and delirium in the intensive care unit. New Eng J Med 2014;370(5):444-54.

[16] Bryczkowski SB, Lopreiato MC, Yonclas PP, Sacca JJ, Mosenthal AC. Risk factors for delirium in older trauma patients admitted to the surgical intensive care unit. J Trauma Acute Care Surg 2014;77(6):944-51.
[17] Robinson TN, Eiseman B. Postoperative delirium in the elderly: Diagnosis and management. Clin Interv Aging 2008;3(2):351-5

[18] Whitlock EL, Vannucci A, Avidan MS. Postoperative delirium. Minerva Anestesiol 2011;77(4):448-56.

[19] Roudsari BS, Psoter KJ, Padia SA, Kogut MJ, Kwan SW. Utilization of angiography and embolization for abdominopelvic trauma: 14 years' experience at a level I trauma center. AJR Am J Roentgenol 2014;202(6):W580-5

[20] Gual N, Inzitari M, Carrizo G, Calle A, Udina C, Yuste A, et al. Delirium Subtypes and Associated Characteristics in Older Patients with Exacerbation of Chronic Conditions. Am J Geriatr Psychiatry 2018;26(12):1204-12.

[21] Marcantonio E, Ta T, Duthie E, Resnick NM. Delirium severity and psychomotor types: their relationship with outcomes after hip fracture repair. J Am Geriatr Soc 2002;50(5):850-7. 